\title{
APLICACIÓN EXPERIMENTAL Y OPERACIONAL DE GIBERELINAS PARA INDUCIR FLORACIÓN EN PINO OREGÓN (Pseudotsuga menziesii (Mirb.) Franco)
}

Braulio Gutiérrez C. ${ }^{1}$

\section{RESUMEN}

Se analiza el efecto de la aplicación de los ácidos giberélicos 4 y 7 (AG4/7) sobre la respuesta floral de injertos de árboles adultos de pino oregón. Se desarrolla una metodología experimental en la que se prueban distintas concentraciones de AG4/7, entre 0 y $1.300 \mathrm{mg} / \mathrm{L}$, dos formas de aplicación y la combinación de AG4/7 con uniconazol.

El tratamiento de mayor efectividad (AG4/7 en concentración de $1.000 \mathrm{mg} / \mathrm{L}$ ) se aplica operacionalmente sobre un huerto semillero clonal de pino oregón y se analiza su efecto sobre la producción de flores del huerto.

Palabras clave: Pino Oregón, Inducción Floral, Giberelinas.

\section{EXPERIMENTAL AND OPERATIONAL APPLICATION OF GIBBERELLIN IN DOUGLAS FIR (Pseudotsuga menziesii (Mirb.) Franco) FLOWERING INDUCTION}

\begin{abstract}
The effect of the application of giberelics acid on the flowering answer of grafts of adult trees of Douglas Fir is analyzed. An experimental methodology which proves different concentrations of $A G 4 / 7$, between 0 and $1.300 \mathrm{mg} / \mathrm{L}$, two forms of application and the combination of $A G 4 / 7$ with uniconazol, is developed.

The best treatment (AG4/7 at $1.000 \mathrm{mg} / \mathrm{L}$ ) is operationally applied on a Douglas Fir clonal seed orchard. The effect of this treatment on the flowers production in the orchard is analyzed.
\end{abstract}

Keywords: Douglas-Fir, Flowering Induction, Gibberellins

\footnotetext{
1 Ingeniero Forestal. Instituto Forestal, Sede Bio Bio. Chile.
}

E-mail: bgutierr@infor.cl 


\section{INTRODUCCION}

Los huertos semilleros son una de las principales fuentes de semilla genéticamente mejorada para la producción de plantas y establecimiento de plantaciones forestales. No obstante lo anterior, es frecuente que en estas unidades la producción de semillas sea escasa, irregular o demande varios años antes de manifestarse en forma constante y homogénea.

Tal situación ha incentivado el desarrollo de técnicas de inducción floral orientadas a favorecer una producción abundante y oportuna de semillas forestales. Entre tales medidas, la aplicación exógena de determinados reguladores de crecimiento ha demostrado ser efectiva, siendo la aplicación de paclobutrazol en eucalipto uno de los resultados más conocidos a nivel nacional.

En pino oregón, y en las coniferas en general, la respuesta floral también puede ser gatillada en forma artificial con reguladores de crecimiento, particularmente con mezclas de ácidos giberélicos (AG), existiendo numerosos antecedentes que indican que las mezclas de AG4 y AG7 (abreviadas como AG4/7) resultan efectivas para promover su floración.

El presente articulo resume los antecedentes generados por el proyecto INNOVA CHILE 02C8FT-01 "Propagación de Genotipos de Interés Comercial de Pino Oregón", ejecutado por el Instituto Forestal, respecto a inducción floral en esta especie. La información entregada corresponde a los resultados obtenidos en ensayos experimentales diseñados para evaluar la respuesta de réplicas injertadas de árboles adultos de pino oregón, ante la aplicación exógena de reguladores de crecimiento, orientados a promover una respuesta en la diferenciación de yemas florales. Incluye también la evaluación de la respuesta floral obtenida después de la aplicación operacional, en un huerto semillero clonal previamente establecido de pino oregón, del mejor tratamiento identificado en los experimentos mencionados.

\section{OBJETIVOS}

Identificar experimentalmente una prescripción concreta de reguladores de crecimiento, con su respectiva metodología de aplicación, para inducir la producción de flores en pino oregón.

Evaluar el resultado de la aplicación operacional de tal metodologia en un huerto semillero clonal de esta especie.

\section{ANTECEDENTES GENERALES}

Pino oregón es una especie monoica diclina (un mismo árbol porta flores unisexuales masculinas y femeninas), cuya floración normalmente se inicia entre los 12 y 15 años de edad, aunque también puede ocurrir a edades inferiores. El ciclo fenológico, desde la formación de las yemas florales hasta la dispersión de las semillas maduras tarda entre 17 y 18 meses (Hermann y Lavender s/f; Leadem s/f y Rose, 2000) 
El proceso de floración se pude dividir en 3 etapas: (i) inducción floral o percepción del estímulo; (ii) citodiferenciación o formación del primordio; y (iii) organogénesis o formación del órgano reproductivo. Las œltimas constituyen la fase de respuesta, que da lugar a la formación de la flor. Respecto a la primera etapa, las señales ambientales (temperatura y fotoperiodo) promueven cambios en las concentraciones hormonales que activan la respuesta floral. De igual forma, la aplicación exógena de reguladores de crecimiento (hormonas sintéticas) también puede estimular a las plantas a florecer.

Efectivamente, desde finales de los ' 70 numerosas publicaciones señalan la eficacia que en general presentan las giberelinas para inducir floración en especies de la familia Pinaceae (Enescu et al., 1977).

Las giberelinas son reguladores de crecimiento encontrados en angiospermas, gimnospermas, helechos, algas y hongos. Se han caracterizado más de 90 tipos de ácidos giberélicos, designados correlativos como AG1, AG2, AG3, E ,AGn de acuerdo al orden cronológico de su aislamiento e identificación (Lozano, s/f).

Las giberelinas promueven crecimiento vegetativo, afirmándose que la floración sólo se promueve cuando las giberelinas no polares (AG4, AG7, AG9, AG15) están presentes en una concentración relativamente alta (Bonet-Masimbert y Doumas, 1992). Entre ellas, el AG4/ 7 ha demostrado ser uno de los más efectivos para inducir floración mediante aplicación exógena, siendo uno de los agentes más efectivos y seguros a la hora de incrementar la floración en huertos semilleros de coniferas.

Al respecto se ha propuesto un modelo hipotético de regulación de la floración en coniferas, donde AG4, sin ser abundante, juega un rol principal. Su efecto seria diferencial dependiendo de si esta se transforma en AG1 para estimular el crecimiento vegetativo, se desactiva a la forma de AG34 o si se fija a un receptor hipotético de AG4 para estimular el crecimiento de órganos reproductivos (Moritz, 1989).

En pino oregón, se ha demostrado que los injertos de material adulto y las plantas de semilla se pueden llevar a una condición de madurez sexual mediante la aplicación exógena de giberelinas, particularmente de AG4/7. Adicionalmente, existen algunas medidas culturales complementarias que pueden potenciar el efecto de la giberelina en la inducción floral, aœn cuando por si solas su efecto sea marginal o nulo para estimular esta respuesta. Entre ellas los tratamientos de poda radicular, anillamiento, y estrés hídrico son los más analizados, señalándose que aparentemente son más efectivos como medida complementaria en plantas jóvenes y que su efecto no es importante en material adulto. Por lo mismo y atendiendo a los negativos efectos que estos pueden tener sobre el vigor y salud de los rametos, su aplicación como medida práctica de manejo no parece recomendable. 
Efectivamente, experimentos mencionados por Ross (1977), que estudió el efecto de aplicaciones de AG4/7 junto a otras medidas para promover la floración (anillamiento, estrés hídrico y fertilización) sobre plantas jóvenes de semillas e injertos de material adulto, le permitieron obtener las siguientes conclusiones:

La respuesta floral es mejor en injertos de material adulto que en plantas juveniles. Siendo promovida significativamente por la giberelina (aumento de hasta tres veces el nœmero de conos por injertos), mientras que los otros tratamientos no fueron significativos por si solos

En injertos los tratamientos de anillamiento, estrés y fertilización disminuyeron la floración. El más antagonista fue el estrés hidrico y el menos la fertilización.

En el caso de las plantas, los tratamientos culturales complementaron y mejoraron el efecto de la hormona, pero como tratamiento individual ninguno fue mejor que la giberelina, incluso la fertilización y el estrés tuvieron un efecto adverso. EI anillamiento promovió la floración pero sólo en un $75 \%$ de lo que logró la hormona sola.

Para efectos de estimular floración, el AG4/7 puede aplicarse como aspersión foliar o inyectarse directamente en el tallo de la planta o injerto. En el primer caso, segœn Pijut (2002) las concentraciones más efectivas fluctœan entre 100 y $600 \mathrm{mg} / \mathrm{L}$ de solución. En el caso de las inyecciones, la absorción por la planta es más directa de modo que las concentraciones son menores; Ross (1977) utiliza $25 \mathrm{mg} / \mathrm{L}$, inyectados en el tallo, justo bajo el comienzo de la copa viva y Greenwood (1977) $50 \mathrm{mg} / \mathrm{L}$, inyectados algunos milímetros bajo la yema terminal.

La aplicación debe efectuarse en primavera, al iniciarse el crecimiento de las yemas vegetativas o durante la elongación activa de los brotes. En cualquier caso, ya sea que la solución se asperje foliarmente o se inyecte, es recomendable repetirla varias veces durante la temporada, ya sea semanal o bisemanalmente. El nœmero de repeticiones que ha sido efectivo varia entre 1 y 11 (Pijut, 2002). Hare (1984) concluye que tres aspersiones bisemanales (200 mg/l) resultan efectivas en Pinus taeda, P. elliottii y P. palustres. Adams et al. (1992) repiten las aspersiones en forma semanal durante 8 oportunidades en Picea mariana, Picea glauca y Pinus banksiana, efectuando la primera de ellas justo al momento de activarse las yemas vegetativas. En pino oregón, Ross (1977) inyecta las giberelinas cada tres semanas, durante tres meses, desde justo antes que se activen las yemas vegetativas. Greenwood (1977) aplica las giberelinas en el mismo periodo, cada 2 semanas, en 5 ocasiones en Pinus taeda. Smith (1998) en Picea mariana la inyecta en el tercio superior de la copa, una sola vez, en la semana siguiente a la activación de las yemas vegetativas, cuando los brotes alcanzan 1 a 2 centímetros de longitud. Wesoly (1987) informa un incremento significativo de flores en Pinus sylvestris con una sola aplicación de $200 \mathrm{mg} / \mathrm{L}$.

No existen mayores antecedentes en cuanto al uso de otros reguladores de crecimiento como complemento a las giberelinas para inducir floración en coníferas. El paclobutrazol (PBZ), que tradicionalmente se utiliza para inducir floración en frutales y eucalipto, ha sido probado en coniferas. Este regulador si bien tiene una acción contraria a las giberelinas, 
actuando como un bloqueador de su síntesis, se ha reportado como efectivo en combinación con AG4/7 para promover respuesta floral en Picea mariana (Smith, 1998). Efectivamente, combinando bajas dosis de PBZ (inyecciones de $1 \mathrm{mg}$ de ingrediente activo) con AG4/7 se obtiene una mayor producción de flores que usando las giberelinas solas. Por su parte el PBZ sin giberelina tiene un efecto muy marginal sobre el incremento de la floración en Picea mariana (op. cit)

\section{MATERIAL Y METODO}

La metodologia empleada comprende dos fases, una experimental efectuada en vivero, para identificar el tratamiento más apropiado para inducir floración en pino oregón y posteriormente, otra operacional en que se evalœa el efecto de tal tratamiento en un huerto semillero clonal de pino oregón.

\section{Fase Experimental}

Se diseñaron cuatro experimentos para definir las dosis más apropiadas de AG4/7 en la estimulación floral de réplicas injertadas de árboles plus adultos de pino oregón. En ellos se prueba una completa bateria de dosis que fluctœan entre 100 y $1.300 \mathrm{mg} / \mathrm{L} \mathrm{de} A G 4 / 7$, aplicado como aspersión foliar, en tres oportunidades, con una frecuencia bisemanal, desde el inicio de la brotación vegetativa. También se evalœa la movilidad del regulador y su efecto en la respuesta floral en función de la zona de aplicación, así como el efecto combinado de AG4/7 con Uniconazol (UCZ) (Cuadro $\mathrm{N}^{\circ} 1$ ).

\section{Cuadro $\mathrm{N}^{\circ} 1$}

DETALLE DE LOS EXPERIMENTOS ESTABLECIDOS

\begin{tabular}{|l|r|rr|}
\hline ENSAYO & & \multicolumn{2}{|c|}{ TRATAMIENTOS(mg/L) } \\
\hline \multirow{3}{*}{$\begin{array}{l}\text { Experimento 1 } \\
\text { Dosis bajas de AG4/7 } \\
\text { (asperjadas a toda la copa). }\end{array}$} & $\mathrm{T} 0$ & 0 & $\mathrm{GA} 4 / 7$ \\
\cline { 2 - 4 } & $\mathrm{T} 1$ & 100 & $\mathrm{GA4} / 7$ \\
\cline { 2 - 4 } & $\mathrm{T} 2$ & 400 & $\mathrm{GA4} / 7$ \\
\cline { 2 - 4 } & $\mathrm{T} 3$ & 700 & $\mathrm{GA4} / 7$ \\
\hline \multirow{2}{*}{$\begin{array}{l}\text { Experimento 2 } \\
\text { Dosis altas de AG4/7 } \\
\text { (asperjadas a toda la copa). }\end{array}$} & $\mathrm{T} 4$ & 0 & $\mathrm{GA4} / 7$ \\
\cline { 2 - 4 } & $\mathrm{T} 5$ & 700 & $\mathrm{GA4} / 7$ \\
\cline { 2 - 4 } & $\mathrm{T} 6$ & 1000 & $\mathrm{GA4} / 7$ \\
\cline { 2 - 4 } & $\mathrm{T} 7$ & 1300 & $\mathrm{GA4} / 7$ \\
\hline \multirow{3}{*}{$\begin{array}{l}\text { Experimento 3 } \\
\text { asperjacación (AG4/7 en una rama) }\end{array}$} & $\mathrm{T} 8$ & 0 & $\mathrm{GA4} / 7$ \\
\cline { 2 - 4 } & $\mathrm{T} 9$ & 700 & $\mathrm{GA4} / 7$ \\
\cline { 2 - 4 } & $\mathrm{T} 10$ & 1000 & $\mathrm{GA4} / 7$ \\
\cline { 2 - 4 } & $\mathrm{T} 11$ & 1300 & $\mathrm{GA4} / 7$ \\
\hline \multirow{2}{*}{$\begin{array}{l}\text { Experimento 4 } \\
\text { AG4/7 más UCZ (AG4/7 } \\
\text { asperjada a toda la copa, UCZ } \\
\text { al sustrato). }\end{array}$} & $\mathrm{T} 12$ & 0 & $\mathrm{GA4} / 7$ \\
\cline { 2 - 4 } & $\mathrm{T} 13$ & $\mathrm{UCZ}+700$ & $\mathrm{GA4} / 7$ \\
\cline { 2 - 4 } & $\mathrm{T} 14$ & 700 & $\mathrm{GA4} / 7$ \\
\cline { 2 - 4 } & $\mathrm{T} 15$ & $\mathrm{UCZ}+1300$ & $\mathrm{GA4} / 7$ \\
\hline
\end{tabular}


Experimento 1. (Dosis bajas): Considera la aplicación, mediante aspersión foliar de tres concentraciones de AG4/7, más un testigo sin aplicación, las cuales se realizan tres veces con una frecuencia bisemanal. Cada tratamiento se aplica sobre 4 clones, representados por un rameto cada uno en un diseño completamente aleatorizado con tres repeticiones.

Experimento 2. (Dosis altas): Se prueban tres concentraciones de AG4/7 más un testigo sin aplicación, usando el mismo esquema de aplicaciones y diseño experimental del experimento 1 .

Experimento 3 (Translocación): Se aplican las mismas concentraciones de giberelina del experimento 2, pero esta vez sobre una sola rama de cada injerto. Cada tratamiento se aplica sobre 2 clones, representados por 4 rametos cada uno, y se repite tres veces en un diseño completamente aleatorizado. La rama que recibe la aplicación se identifica con una etiqueta para su posterior evaluación.

Experimento 4. (AG4/7 + UCZ): Se usa el mismo esquema de aplicaciones y diseño del experimento 1. Las aplicaciones de AG4/7 se realizan en forma bisemanal ( 3 aplicaciones); UCZ se aplica al sustrato por una vez, en dosis de $0,25 \mathrm{~g}$ de ingrediente activo por cada centímetro de perímetro en el cuello del injerto.

Para establecer los ensayos se usó injertos de árboles adultos efectuados con dos años de anterioridad. Estos fueron manejados en macetas de polietileno de 5 litros de capacidad, fertilizados con productos de liberación controlada (Osmocote 19:6:10, a razón de $12 \mathrm{~g} /$ planta) y mantenidos en vivero hasta el momento de establecer los distintos ensayos de inducción floral en primavera de 2003.

Los reguladores de crecimiento utilizados para estimular la inducción floral fueron los siguientes:

Giberelinas: Se usó una mezcla de los ácidos giberélicos AG4 y AG7 en el producto comercial ProVide ( $2 \%$ i.a.), el que se aplicó como aspersión foliar en soluciones de distinta concentración, junto con el surfactante HIPOINT, a razón de $0,25 \mathrm{ml} / \mathrm{L}$.

Antigiberelinas: Se usó Uniconazol (UCZ) a través del producto comercial SUNNY (50 $\mathrm{g} / \mathrm{L}$ i.a.). El uniconazol (UCZ) es un isómero del paclobutrazol (PBZ), al igual que este, corresponde a una antigiberelina del grupo de los triazoles. El uniconazol se aplicó en solución, directamente a las raices de los injertos, a razón de $0,25 \mathrm{~g}$ de ingrediente activo por cada centímetro de perímetro en el cuello de los injertos.

En los injertos de los experimentos 1, 2 y 4 se seleccionó tres ramas (el lider más dos laterales) las cuales se identificó con etiquetas, para hacer un seguimiento de su elongación, nœmero y tipo de yemas. Lo mismo se hizo con la rama de cada planta que recibió aplicación de AG4/7 del experimento 3 . 
Para la evaluación de los experimentos se efectuó tres mediciones. La primera de ellas al momento de montar cada ensayo (primavera 2003), midiéndose la longitud de las ramas individualizadas en cada planta y el nœmero de yemas vegetativas presentes en ellas.

La segunda medición se realizó en otoño del 2004, una vez terminada la temporada de crecimiento vegetativo. Se registró la longitud de las ramas y el número de yemas vegetativas formadas durante la temporada de crecimiento. Su objetivo fue determinar los incrementos producidos en ambas variables y relacionarlos con los tratamientos aplicados.

Este procedimiento constituye una forma de estimar anticipadamente el efecto de las aplicaciones de giberelinas, por cuanto uno de sus efectos secundarios más evidente es la mayor elongación de las ramas y la proliferación o aumento en el número de yemas totales, mientras que el efecto sobre la repuesta floral sólo se presenta en la temporada de floración del año siguiente.

La tercera medición se efectuó a comienzos de primavera del año 2004 (octubre, 2004), periodo en el cual se manifiesta la floración de los primordios florales formados en la temporada anterior. En esta evaluación se registró el número de flores masculinas y femeninas.

Las comparaciones entre tratamientos de un mismo ensayo, así como entre ensayos por medio de tratamientos comunes que actúan como conectores, permitió verificar el efecto sobre la respuesta floral de las distintas dosis de reguladores de crecimiento empleados.

El contraste de los tratamientos del experimento 3 con su respectivo testigo y con los tratamientos del experimento 2 permitió verificar si el efecto de la aplicación es local en la rama tratada o si se expande a toda la planta.

\section{Fase Operacional}

Correspondió a la aplicación operacional del mejor tratamiento identificado en la etapa experimental. Esta se efectuó sobre un huerto semillero clonal de pino oregón, establecido el año 2002, como una iniciativa conjunta de INFOR Y CONAF, en el sector Huertos Familiares,en el Km 28 del camino Temuco-Nueva Imperial, Región de La araucania. Tal huerto posee una superficie de 1,7 hectáreas, donde se representan 42 clones, distribuidos en 20 bloques completos al azar. 19 bloques recibieron el tratamiento de inducción floral y uno se dejó como testigo para efecto de las comparaciones.

Considerando que esta aplicación no constituye un ensayo, sino que una medida de manejo operacional, para efectos dè la evaluación se efectuó una comparación simple entre la respuesta floral observada en dos bloques del huerto; por una parte el bloque testigo sin aplicación de giberelinas y por otra, un bloque escogido al azar de entre todos los que recibieron el tratamiento de inducción.

En ambos bloques se monitoreó todos los rametos existentes contabilizando el número de flores masculinas y femeninas de cada uno, así como la altura individual de cada rameto. Adicionalmente en el resto del huerto se hizo una contabilización general de todos los rametos, 
clasificándolos en función de la abundancia de flores exhibidas. Las categorias empleadas fueron: escasas ( 1 a 5 flores); medias ( 6 a 20 flores); y abundantes (más de 20 flores). La distribución de los rametos en cada categoria se comparó con la del bloque testigo sin aplicación.

\section{RESULTADOS Y DISCUSIÓN}

\section{Fase Experimental}

En la Figura $\mathrm{N}^{\circ} 1$ se representa la relación entre la respuesta floral (en términos del porcentaje de plantas evaluadas que presentó flores y el número de flores por planta evaluada) respecto a los parámetros predictivos del efecto secundario de las giberelinas (incremento en longitud de ramas e incremento en número de yemas totales (vegetativas y florales)). En el gráfico los tratamientos del eje $\mathrm{X}$ (abscisas) se ordenan de menor a mayor concentración de AG4/7 (Cuadro $N^{\circ} 1$ ).

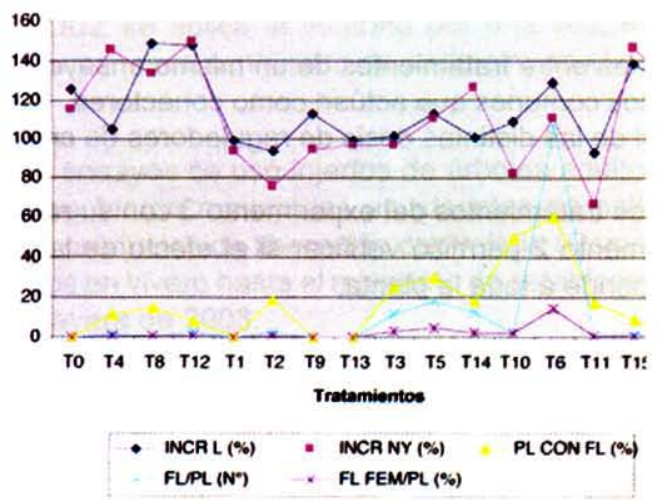

Respuesta floral expresada en porcentaje de plantas con flores en cada tratamiento (PL CON FL), $\mathrm{N}^{\circ}$ de flores por planta evaluada en cada tratamiento (FL/PL) y $\mathrm{N}^{\circ}$ de flores femeninas por planta evaluada en cada tratamiento (FL FEM/PL).

RELACION ENTRE LOS PARAMETROS D RAMAS (INCR L) E INCREMENTO EN NÚMERO DE YEMAS (INCR NY).

En términos generales, la relación entre la elongación de las ramas y el aumento del número de yemas exhiben una tendencia similar y que no coincide con las concentraciones de AG4/7. Por otra parte, su relación con la respuesta floral no es evidente. Si se considera que uno de los efectos de las giberelinas es promover el crecimiento, se hubiese esperado que a mayores incrementos en largo de ramas y número de yemas, se expresase también una mejor respuesta en formación de flores. 
Respecto a los parámetros de respuesta floral, existe una tendencia a que estos mejoren al incrementar la concentración de AG4/7 hasta los $1.000 \mathrm{mg}$ (T6) y volver a decrecer a concentraciones mayores. Salvo algunas excepciones (T1, T9, T13 y T7) los tratamientos con aplicación de reguladores muestran una mejor respuesta floral que los testigos sin aplicación (T0, T4, T8, T12).

La situación anterior es relativamente coincidente con la publicada por Ross (1977), quien afirma que aunque la magnitud del efecto de las giberelinas sobre la inducción floral es variable, en injertos de pino oregón, el nœmero de plantas que produce flores puede ser hasta 8 veces mayor en plantas tratadas con giberelinas respecto a las testigos. En estos experimentos tal diferencia se produjo sólo en el tratamiento T6, que indujo casi 6 veces más plantas con flores que el promedio de los testigos sin regulador.

Al respecto, aún cuando la mayoría de los tratamientos no induce una respuesta considerablemente superior a los testigos, es destacable el comportamiento exhibido por el tratamiento T6 (1.000 mg AG4/7) que se diferencia favorablemente de los testigos y restantes tratamientos ensayados. No obstante, la magnitud de esta dosis es considerablemente superior a las de 100 a 600 mg/L, señaladas por Pijut (2002) como las mas efectivas para inducir floración.

El análisis especifico de cada experimento es presentado a continuación.

\section{Experimento 1: Dosis Bajas AG4/7}

Los tratamientos T2 y T3, equivalentes a concentraciones de AG4/7 de 400 y 700 mg/ $\mathrm{L}$, producen una manifestación floral superior al testigo sin aplicación (TO), que no produjo flores. A su vez, el tratamiento T1 (100 mg/L) tampoco tuvo efectos sobre la floración.

Estos resultados permiten definir el rango inferior de la concentración de giberelinas, cuando se aplican para estimular floración. Las concentraciones más bajas (T1) no tienen efecto sobre este fenómeno, el cual se intensifica al aumentar la concentración en T2 y T3.

Al combinar estos resultados con los obtenidos en el experimento de dosis altas se observa una tendencia creciente en la respuesta floral que sigue aumentando al usar concentraciones mayores de giberelinas, encontrándose su óptimo alrededor de los 1.000 $\mathrm{mg} / \mathrm{L}$ en el tratamiento T6 del experimento 2 , para posteriormente decrecer en $\mathrm{T} 7$ al usar una concentración superior $(1.300 \mathrm{mg} / \mathrm{L})$.

\section{Experimento 2: Dosis altas AG4/7}

En relación al testigo (T4) todos los tratamientos de altas dosis de AG4/7 (T5, T6 y T7) exhiben un mayor incremento en la longitud de las ramas y un menor incremento en el número de yemas, aunque no todas estas diferencias son estadisticamente significativas.

En términos de respuesta floral se aprecia una clara diferencia entre tratamientos. T5 (700 mg/L AG4/7) y T6 (1.000 mg/L AG4/7) son claramente superiores al testigo, mientras que 
$\mathrm{T} 7$ (1.300 mg/L AG4/7) no exhibe respuesta floral. Los mejores resultados se obtienen con el tratamiento T6 que logra inducir la formación de flores en el $60 \%$ de las plantas evaluadas y un promedio de más de 100 flores por planta, mayoritariamente masculinas, pero también con la mayor cantidad de flores femeninas de todos los tratamientos ensayados.

Por otra parte, $T 7(1.300 \mathrm{mg} / \mathrm{L})$ además de no producir ninguna respuesta floral exhibió una mayor mortalidad de plantas, indicando una posible toxicidad del producto en la concentración aplicada y confirmando que los valores óptimos se encuentran cercanos a los $1.000 \mathrm{mg} / \mathrm{L}$.

Si bien en este experimento T7 no tuvo efecto sobre la floración, se observa que al usar la misma concentración de regulador de crecimiento, pero aplicado en forma local (experimento 3 ) o combinado con uniconazol (experimento 4), se produce un ligero efecto sobre la floración. Este efecto, aunque estadisticamente no es diferente de los testigos, muestra que los procedimientos que neutralizan o reducen a la giberelina absorbida por la planta, permiten que dosis excesivas se reduzcan a otras que pueden provocar efecto sobre la floración.

\section{Experimento 3: Translocación AG4/7}

En este experimento se observa que sólo $T 10(1.000 \mathrm{mg} / \mathrm{L})$ induce una respuesta floral significativamente superior al testigo (T8), mientras que los tratamientos restantes, aunque no se diferencian estadísticamente del testigo, muestran valores que son inferiores a los de él.

El comportamiento de T10 es consistente con el observado en el tratamiento T6 del experimento 2, en término del número de plantas que formaron flores, sin embargo el número de flores por planta fue bastante más reducido al aplicar el producto en forma local sobre una sola rama (T10, experimento 3 ), que al asperjarlo en toda la copa (T6, experimento 2 ).

La dosis de $700 \mathrm{mg} / \mathrm{L}$ de AG4/7 (T3, T5 y T14 de los experimentos 1, 2 y 4 respectivamente) que al asperjarlo en toda la copa tiene un importante efecto inductor de flores, al aplicarla localmente sobre una sola rama (T9) no produce efecto alguno. Coincidentemente, en el T13 del experimento 4, donde también se usan $700 \mathrm{mg} / \mathrm{L}$ de giberelinas, pero en combinación con UCZ, tampoco exhibe efecto sobre la floración.

El hecho de que la dosis de $700 \mathrm{mg} / \mathrm{L}$ sea efectiva en todos los casos en que se aplica pura, sobre toda la copa y no tenga efecto cuando se aplica en forma local, o combinada con UCZ, parece señalar que la cantidad de producto que se absorbe o que actúa en la planta se ve reducida como consecuencia de la aplicación local o el efecto antagónico del UCZ.

A la inversa, pero coincidiendo con lo anterior, el tratamiento T11 de $1.300 \mathrm{mg} / \mathrm{L}$ de giberelina, si bien no se diferencia estadisticamente del testigo, sí presenta una ligera respuesta floral al aplicarlo en forma local sobre una rama. Sin embargo, al asperjar la misma dosis sobre toda la copa (T7) no produce efecto en la floración y se asocia a una mayor mortalidad de plantas, presumiéndose una probable toxicidad (experimento 2). En este mismo sentido, si se revisan los resultados del tratamiento T15 en el experimento 4, donde también se usan 1.300 $\mathrm{mg} / \mathrm{L}$ de giberelina, pero combinados con UCZ, se vuelve a encontrar un ligero efecto sobre la floración, aunque otra vez no hay diferencias significativas con su respectivo testigo (T12). 
El comportamiento del tratamiento de $1.300 \mathrm{mg} / \mathrm{L}$ de giberelina se puede interpretar en forma equivalente al de $700 \mathrm{mg} / \mathrm{L}$. En este caso la combinación con UCZ, o la aplicación en forma local, reducirian la cantidad de producto que se absorbe o que actúa en la planta. De esta forma, una dosis que aparentemente puede ser demasiado alta al aplicarla directamente sobre toda la copa, se reduce a un nivel menor que le permite manifestar algún grado de efecto sobre la floración.

Recapitulando, el menor efecto de la dosis de $1.000 \mathrm{mg} / \mathrm{L}$ de $A G 4 / 7$ al aplicarlo en forma local (T10) respecto a su aspersión en toda la copa (T6), sumado al menor rendimiento de los restantes tratamientos del experimento 3 respecto de su testigo (T8), permiten concluir que la aplicación de AG4/7 localizada en una sola rama, no resulta efectiva, en las dosis ensayadas, para promover floración en injertos de pino oregón.

La respuesta observada en este experimento, no puede atribuirse directamente a la movilidad de la hormona. Aparentemente el menor efecto es sólo consecuencia de la menor cantidad de producto que es efectivamente absorbido por la planta.

\section{Experimento 4: Interacción AG4/7 y UCZ}

Los resultados de este experimento indican que los tratamientos mixtos, que combinan AG4/7 y UCZ tienen un efecto inferior o no significativamente distinto al del testigo sin aplicación (T12).

Efectivamente entre los tratamientos mixtos, el tratamiento T13 $(700 \mathrm{mg} / \mathrm{l}$ de AG4/7 + UCZ) no indujo ninguna respuesta floral. El tratamiento T15 (1.300 mg/L de AG4/7 + UCZ) produjo una respuesta que no se diferencia de la del testigo.

Por su parte, el tratamiento de giberelina pura, correspondiente a T14 $(700 \mathrm{mg} / \mathrm{L}$ de AG4/7), resulta claramente superior al testigo y los tratamientos mixtos. A su vez su comportamiento resulta consistente con el expresado por los tratamientos T3 y T5 de los experimentos 1 y 2 respectivamente, que corresponden a la misma dosis de AG4/7.

El efecto antagónico de las giberelinas y la antigiberelina UCZ parece producir una neutralización de las primeras que explicaria los resultados obtenidos. Así, en el caso del tratamiento mixto con menor dosis de AG4/7 (T13; $700 \mathrm{mg} / \mathrm{L}+\mathrm{UCZ}$ ) el uniconazol evita la expresión del efecto de las giberelinas, $y$ a diferencia de los resultados experimentados por esta concentración de AG4/7 en los otros experimentos, en este caso no produce efecto alguno sobre la floración. A su vez, en el caso de T15, con una concentración de $1.300 \mathrm{mg} / \mathrm{L}$ de AG4/ 7 , el uniconazol neutraliza una fracción de las giberelinas, haciendo un efecto similar a usar una dosis menor de ellas.

Contribuye a respaldar la interpretación anterior, el hecho de que en el experimento 2 el tratamiento T7 que también correspondia a una concentración de AG4/7 de $1.300 \mathrm{mg} / \mathrm{L}$ no produjo respuesta floral y evidenció la menor sobrevivencia de plantas, situación que se atribuye a una eventual toxicidad del producto en esa concentración. Sin embargo, al usar la misma concentración, pero mezclada con UCZ se produce una respuesta floral mejor que en el tratamiento puro. En este caso, la situación es coincidente con la señalada por Smith (1998) 
quien reporta que la aplicación de PBZ (isómero del UCZ) mejora el efecto de la giberelina sola.

Ante la interpretación de estos resultados, parece lógico pensar que el efecto del UCZ mejora la acción de las giberelinas cuando estas se aplican en concentraciones superiores a las óptimas. Consecuentemente, resulta un sistema más sencillo y barato utilizar sólo giberelinas, en dosis cercanas a $1.000 \mathrm{mg} / \mathrm{L}$, en vez de dosis mayores complementadas con UCZ.

\section{Fase Operacional}

Se aplicó el tratamiento T6 de $1.000 \mathrm{mg} / \mathrm{L}$ de AG4/7 a 19 de los 20 bloques que componen el huerto semillero clonal descrito en el apartado de metodologia.

Tal como se podía esperar de los resultados obtenidos en la fase experimental, los rametos tratados con giberelina exhiben una mayor floración que el testigo. No obstante, atendiendo a que la aplicación en el huerto no es un ensayo con repeticiones ni diseño estadistico, no se puede evaluar la significancia estadística de esta diferencia. Aún así, esta parece de escasa magnitud y menor a la observada en los ensayos experimentales.

El porcentaje de rametos que exhibe flores es relativamente similar en el testigo y en los bloques tratados. En ambos casos el nú mero de flores masculinas y el número de rametos que las portan, son mayores que para las femeninas.

El número total de flores es superior en los árboles tratados que en los sin tratar. Particularmente el número de flores femeninas aumenta en relación al testigo, el cual prácticamente no las evidencia. Este aspecto es relevante, por cuanto es de estas flores de la que depende fundamentalmente la producción de semillas.

En el Cuadro $\mathrm{N}^{\circ} 2$ se resume los antecedentes de la comparación entre el bloque testigo y un bloque tratado escogido al azar dentro del huerto. En el se presentan algunas relaciones promedios en términos de porcentaje de rametos con flores, número de flores por rameto y número de flores por metro de copa. 


\section{Cuadro $\mathrm{N}^{\circ} 2$ \\ RESPUESTA FLORAL ANTE APLICACIÓN DE GIBERELINAS EN UN HUERTO SEMILLERO CLONAL DE PINO OREGÓN}

\begin{tabular}{|l|c|c|}
\hline Variable & $\begin{array}{c}\text { Testigo } \\
\text { (sin aplicación) }\end{array}$ & $\begin{array}{c}\text { AG4/7 } \\
\text { (1.000 } \mathbf{~ m g / L )}\end{array}$ \\
\hline $\mathbf{N}^{\circ}$ de rametos evaluados & $\mathbf{3 3}$ & $\mathbf{3 4}$ \\
•Altura promedio & 2,39 & 2,54 \\
\hline $\mathbf{N}^{\circ}$ Total de flores observadas & $\mathbf{9 2 5}$ & $\mathbf{1 . 4 7 8}$ \\
-Masculinas & 924 & 1.263 \\
- Femeninas & 1 & 215 \\
\hline Rametos con flores (\%) & $\mathbf{7 2 , 7}$ & $\mathbf{7 9 , 4}$ \\
-Masculinas & 72.7 & 79.4 \\
Femeninas & 3.0 & 8.8 \\
\hline $\mathbf{N}^{\circ}$ promedio de flores por rameto & $\mathbf{2 8 , 0 3}$ & $\mathbf{4 3 , 4 7}$ \\
-Masculinas & 28,00 & 37,15 \\
-Femeninas & 0,03 & 6,32 \\
\hline $\mathbf{N}^{\circ}$ promedio de flores por metro de copa & $\mathbf{1 1 , 7 3}$ & $\mathbf{1 7 , 1 3}$ \\
-Masculinas & 11,72 & 14,64 \\
-Femeninas & 0,01 & 2,49 \\
\hline
\end{tabular}

Respecto a la abundancia de flores por rametos, en los árboles tratados aumenta la proporción de individuos que presentan categorias superiores de abundancia de flores femeninas. En efecto, en el testigo los rametos que portan flores femeninas sólo las presentan en forma escasa, en cambio en los que recibieron la aplicación de giberelina las portan en forma abundante o media. En el caso de las flores masculinas, la distribución es relativamente similar para los rametos tratados que para los testigos (Figuras $\mathrm{N}^{\circ} \mathrm{s} 2$ y 3 ).

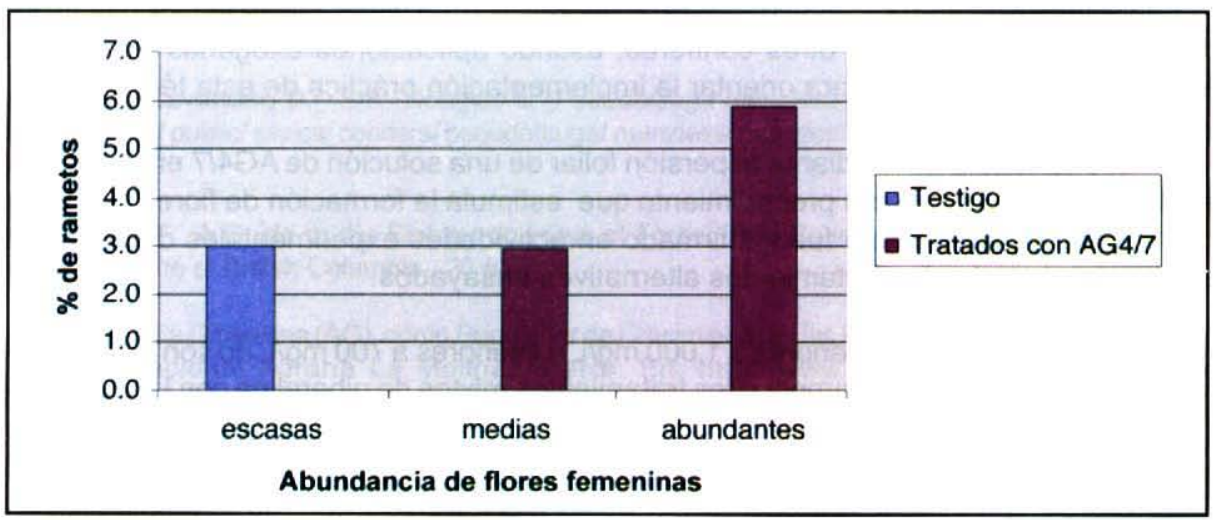

Figura $N^{\circ} 2$

DISTRIBUCIÓN DE RAMETOS SEGÚN ABUNDANCIA DE FLORES FEMENINAS 


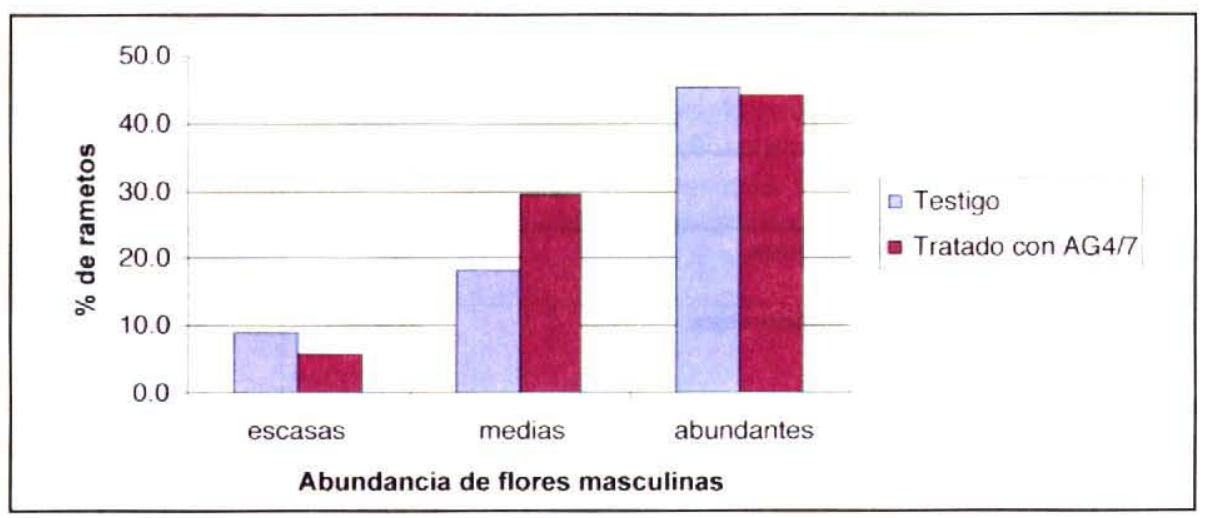

Figura $N^{\circ} 2$

\section{DISTRIBUCIÓN DE RAMETOS SEGÚN ABUNDANCIA DE FLORES FEMENINAS}

Como resultado general se observa una incidencia positiva de la aplicación de giberelinas en todos los parámetros que cuantifican la floración del huerto. Aœn asi, en términos absolutos, la producción de flores continúa siendo escasa para el objetivo de producir semillas en forma abundante. En cualquier caso, esta situación parece derivarse del incipiente estado de desarrollo del huerto, más que del tratamiento de inducción floral empleado. Por lo mismo, resultará interesante repetirlo cuando este tenga más edad y sus rametos hayan desarrollado una mayor altura y volumen de copa.

\section{CONCLUSIONES}

Existen abundantes antecedentes bibliográficos que confirman la factibilidad de inducir floración en pino oregón, y otras coniferas, usando aplicaciones exógenas de giberelinas, estos resultan adecuados para orientar la implementación práctica de esta técnica.

Las aplicaciones mediante aspersión foliar de una solución de AG4/7 en concentración de $1.000 \mathrm{mg} / \mathrm{L}$ constituye un procedimiento que estimula la formación de flores en injertos de pino oregón. Este resultado fue confirmado en actividades experimentales donde demostró ser el mejor de todos los tratamientos alternativos ensayados.

Concentraciones superiores a $1.000 \mathrm{mg} / \mathrm{L}$ e inferiores a $700 \mathrm{mg} / \mathrm{L}$ no son recomendables para este efecto. Así como tampoco los tratamientos mixtos de giberelina con UCZ, ni aquellos en que la hormona se asperja en forma local sobre una reducida fracción de la copa.

A nivel operacional la aplicación de $1.000 \mathrm{mg} / \mathrm{L}$ de $\mathrm{AG} 4 / 7$ en un huerto clonal de pino oregón logró inducir niveles de floración superiores a las de un testigo sin aplicación. Aún así, debido al escaso desarrollo de los rametos del huerto, el efecto no fue suficiente como para generar una producción masiva de semillas. 
La utilidad de la aplicación exógena de giberelinas para promover la floración y consecuentemente incrementar la producción de semillas genéticamente mejoradas en huertos semilleros de pino oregón es muy promisoria.

\section{RECONOCIMIENTOS}

El autor desea agradecer a los Sres. Patricio Alzugaray y Jaime Espejo por la colaboración prestada en distintas fases de la definición y montaje de los ensayos, así como a los Sres. Marco Barrientos y Nabor Barriles por su colaboración en la aplicación operacional del tratamiento de inducción floral en el huerto clonal de pino oregón.

\section{REFERENCIAS}

Adams, G. Irving, J. y Greenwood, M., 1992. Optimization of Environmental Regimes for Flowering in an Indoor Breeding Hall for Black, White Spruce and Jack Pine. En: Proceedings of Symposium IUFRO AFOCEL Mass Production Technology for Genetically Improved Fast Growing Forest Tree Species. Bordeaux, France. 14-18 September, 1992. Pp:219-277.

Bonet-Masimbert, M. y Doumas, P., 1992. Physiological Approach to Flowering Induction in Conifers. En: Proceedings of Symposium IUFRO AFOCEL Mass Production Technology for Genetically Improved Fast Growing Forest Tree Species. Bordeaux, France. 14-18 September, 1992. Pp:181-188.

Enescu, V.; Popescu, P. y Contescu, L., 1977. Blossoming Artificial Inducing in a Clonal Orchard of Pseudotsuga menziesii. En: Third World Consultation on Forest Tree Breeding. Canberra, Australia 21 al 26 de Marzo de 1977. Pp:1071-1078.

Greenwood, M., 1977. Flower Stimulation Techniques for Loblolly Pine, Pinus taeda. En: Third World Consultation on Forest Tree Breeding. Canberra, Australia, 21 al 26 de marzo de 1977. Pp:1031- 1042.

Hare, R., 1984. Application Method and Timing of Gibberellin A4/7 Treatments for Increasing Pollen Conebud Production in Southern Pines. Can Jour of For. Res. 14:123-131.

Hermann, R. y Lavender, D., S/F. Douglas-fir (Pseudotsuga menziesii (Mirb.) Franco). En: http://www. Forestworld. com/ public/ silvics/ conifers/ pseudotsuga/ menziesii/ menziesii_c1.html. (Consulta, Septiembre 2003).

Leadem, C., S/F. A Guide to the Biology and Use of Forest Tree Seeds. Ministry of Forest Research Program. Province of British Columbia. $20 \mathrm{p}$.

Lozano, K., S/F. La Giberelina (AG), como Regulador de Crecimiento en las Plantas. Facultad de Agronomia, Universidad Nacional Agraria La Molina. Perœ. En: http://www.lamolina.edu.pe/FACULTAD/ Agronomiahorticultura/propagacion/fitohormonas/ klozano.doc (Consulta, Mayo 2004)

Moritz, T., 1989. Gibberellins and Flower Bud Differentiation in Spruce. The Swedish Univ. Agric. Sci. Dept. Forest. Genet. \&Plant physiology. ISBN 91-576-4007-6.

Pijut, P., 2002. Eastern White Pine (Pinus strobus) Flowering in Response to Spray Application of Gibberillin A4/7 or Procone. North. Jour. Appl. For. 19(2):68-72. 
Rose, R., 2000. Silviculture: Reforestation. Class Notes (FOR 442). Department of Forest Resources. College of Forestry. Oregon State University.

Ross, D., 1977. Influences of Gibberellins and Cultural Practices on Early Flowering of Douglas-Fir Seedlings and Grafts. En: Third World Consultation on Forest Tree Breeding. Canberra, Australia 21 al 26 de marzo de 1977. Pp:997-1008.

Smith, R., 1998. Effects of Stem Injections of Gibberellins A4/7 and Paclobutrazol on Sex Expression and the Within-Crown Distribution of Seed and Pollen Cones in Black Spruce (Picea mariana). En: Canadian Journal of Forestry Research 28: 641-651.

Wesoly, W., 1987. Effect of Spraying with Gibberellin A4/7 on Flowering and Endogenous Gibberellin Content in Buds of Pinus sylvestris Grafts. Forest Ecology and Management. 19(1-4):121-127. 